\title{
Does retinal size have a unique correlate in perceived size?
}

\author{
ALI A. LANDAUER, 1,2 UNIVERSITY OF WESTERN AUSTRALIA \\ WILLIAM EPSTEIN, ${ }^{3}$ UNIVERSITY OF WISCONSIN, MADISON
}

\begin{abstract}
Subjects estimated the size and distance of a single electroluminescent disc in the absence of distance cues and without the use of visual comparators. For different groups of Ss the disc subtended a visual angle of $1,2,4$, or 8 deg. The size estimates varied directly with visual angle and the distance estimates varied inversely with visual angle. These results were considered in relation to the question of whether or not retinal size has a direct correlate in perceived size.
\end{abstract}

Does retinal size have a unique perceived-size correlate? Specifically, can evidence be adduced that variations of visual angle are accompanied by variations of perceived size, irrespective of concomitant variations of perceived distance? This question has been examined by Wallach and McKenna (1960), Rock and McDermott (1964), and Gogel (in press) with contradictory results.

The present experiment was also directed to this question. The plan was to secure size estimates of a single nonrepresentative target, presented in a totally dark field, without any accompanying visual targets or any distance cues. Consider the following possible outcomes of an experiment in which size and distance judgments are obtained of identically shaped, nonrepresentative targets that subtend different visual angles, under the conditions described.

(1) Judged size remains constant and judged distance varies inversely with visual angle.

(2) Judged size varies directly with visual angle and perceived distance remains constant.

Outcomes 1 and 2 represent instances of size-distance invariance. They provide no evidence that visual angle has a unique correlate in perceived size, independently of perceived distance. Instead, these outcomes indicate size-distance dependence.

(3) Judged size and judged distance remain constant over variations of visual angle.

Outcome 3 provides no evidence of a size-distance interaction of the sort required by the size-distance invariance hypothesis. Neither would there be evidence that visual angle and perceived size are uniquely correlated.

(4) Judged size varies directly with visual angle and judged distance varies unsystematically with variations of visual angle.

(5) Judged size varies directly with visual angle and judged distance varies inversely with visual angle.

Outcome 4 would provide unequivocal evidence of direct determination of perceived size by visual angle. Outcome 5 cannot be interpreted simply and we will consider this outcome in detail later.

In an earlier study (Epstein \& Landauer, 1969), magnitude estimations of size and distance of a variable relative to a modulus were obtained in the absence of distance cues. Estimates were provided by different groups under three conditions: (1) physical size and distance variant, visual angle of the variable constant and equal to the module, (2) physical size constant, physical distance and visual angle of variable changing, and (3) physical distance constant, physical size and visual angle of the variable changing. The results under Conditions 2 and 3 were highly similar. In each case both size and distance estimates conformed to relative visual angle. Outcome 5 was obtained. The results strongly imply that relative visual angle has a perceived relative-size correlate independent of perceived relative distance. But the experiment does not settle the question of whether or not a single retinal subtense has a unique correlate in perceived absolute size. The present experiment was designed to answer this question.

\section{Apparatus and Stimuli}

\section{METHOD}

The standard was an electroluminescent disc, $82 \mathrm{~mm}$ diam, presented in total darkness. The standard was at eye level straight ahead of $S$. For different Ss the standard was located at the following distances: $59,118,235,470 \mathrm{~cm}$. At these distances the standard subtended a visual angle of $8,4,2$, and $1 \mathrm{deg}$, respectively. At each distance the disc's luminance was adjusted by a variac so that brightness was subjectively equal at all distances. A combination head-chin rest was used to eliminate gross head movement. An occluder in front of the left eye confined vision to the right eye.

\section{Subjects}

Four groups of $20 \mathrm{Ss}$ with uncorrected vision were used, a different group for each visual angle. The Ss were students in an introductory course in psychology, who had volunteered to fulfill a course requirement. There were 40 men and 40 women, ranging in age from 18 to 30 years (mean 20.4).

\section{Procedure}

The Ss were assigned randomly to one of the four experimental groups. The $S$ was met in a waiting-room situated on a different floor than the laboratory. There, $S$ was blindfolded and led to the experimental room, where he was seated and the blindfold was removed in total darkness. This procedure was adopted to prevent $S$ from obtaining any information about the size of the laboratory and to control S's assumptions about the possible size and distance of the disc (Epstein, 1967; Gogel, 1968). Blindfolding also helped to reduce the time in the laboratory necessary for dark adaptation. In the laboratory $S$ remained in total darkness for $5 \mathrm{~min}$ prior to the initiation of testing.

Table 1

Items on the Equal Step Size and Distance Scales on which Ss Located Their Judgments. The Scale Values in Parentheses $(\mathrm{mm}$ for size, $\mathrm{cm}$ for distance) Did not Appear on the Scale Shown to S.

\begin{tabular}{|c|c|}
\hline Size Judgments & Distance Judgments \\
\hline $\begin{array}{l}\text { Pin Head (1) } \\
\text { Nail Head (4) } \\
\text { Small Shirt or Blouse Button (8) } \\
\text { Dime (17) } \\
\text { Quarter (22) } \\
\text { Half Dollar (31) } \\
\text { Ping-Pong Ball or Golf Ball (43) } \\
\text { Tennis Ball (64) } \\
\text { Baseball (73) } \\
\text { Large Grapefuit (130) } \\
\text { Bowling or Volley Ball (220) } \\
\text { Large L.P. Record (305) } \\
\text { Car Wheel (450) } \\
\text { Bus Wheel (900) }\end{array}$ & $\begin{array}{l}\text { Length of Finger (8) } \\
\text { Span of Hand (18) } \\
\text { Length of a 12-in. Ruler ( } 31) \\
\text { Length of Extended Arm }(65) \\
\text { Span of Both Arms Extended (140) } \\
\text { Length of a Bed (200) } \\
\text { Length of Standard Chevrolet Car (350) } \\
\text { Length of Large Station Wagon (500) } \\
\text { Length of a Bus (1100) } \\
\text { Width of State Street }(1600) \\
\text { Width of a Football Field }(4900) \\
\text { Length of a Football Field }(9200)\end{array}$ \\
\hline
\end{tabular}




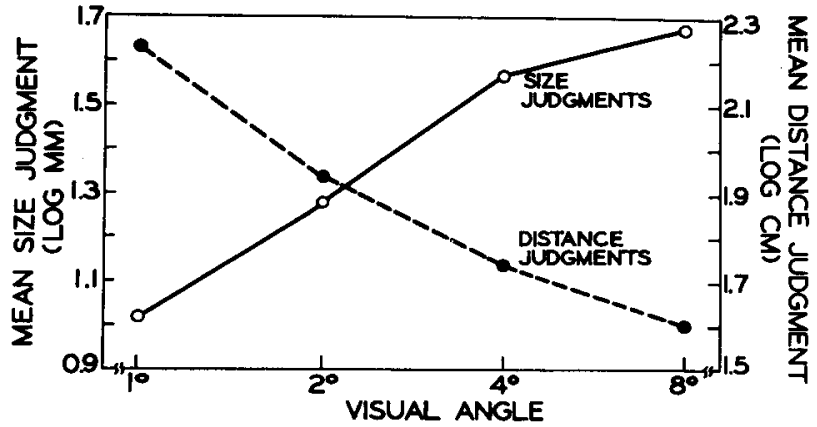

Fig. 1. The size and distance judgments of the standard disc, logarithmically transformed.

The $S$ was informed that he would see a dimly illuminated disc straight ahead, and that he would be required to judge its size and its distance away from him. The instructions stressed that $S$ should judge size and distance at the same time. Half the number of $S s$ in each group were asked to report their size judgment first, while the other half were instructed to report their distance judgment first. The instructions encouraged $S$ to make the judgment in terms of well known objects and popular distances; to report the size "to be about the same size as an $X$..." and to report distance "... to be about the same distance away as the length of a Y ..." No hints were given regarding size or distance of the disc, and any questions about these two variables were deferred. When $S$ indicated that he understood the task, the disc was activated and remained illuminated until $S$ responded. Each $S$ supplied only one size and one distance judgment for a single disc. The judgments of three Ss who gave astronomical responses (e.g., "the same distance as the moon") were discarded and they were replaced by different Ss.

After completing his judgments, $S$ was blindfolded again, in darkness, and led to another room where the blindfold was removed. He was shown two scales that were marked off in equal intervals (see Table 1), and he was instructed to locate his judgment on the scale by placing a mark on each continuum. This procedure was particularly helpful in identifying idiosyncratic responses and responses that referred to sizes or distances that are normally variable, e.g., "a large dinner plate," "a pencil's length," "the length of my room."

\section{RESULTS}

The size and distance judgments were assigned metric equivalents by applying the scale values shown in Table 1 . For example, if $S$ reported that the disc was the size of a dime at a distance equal to the size of an extended arm, the values $17 \mathrm{~mm}$ and $65 \mathrm{~cm}$ were assigned to the size and distance, respectively, These metric values were transformed logarithmically. The results are plotted in Fig. 1.

Figure 1 shows that judged size tended to vary directly with visual angle and judged distance tended to vary inversely with visual angle. Separate analyses of variance of the logarithmically transformed data showed significant overall main effects of visual angle both for size and distance judgments: size, $F(3,72)=8.37$, $\mathrm{p}<.001$; distance, $\mathrm{F}(3,72)=4.65, \mathrm{p}<.01$. Neither order of judgment, whether size or distance was reported first, nor the interaction between visual angle and order of judgment was significant.

The visual angle equivalents of the paired size and distance judgments were computed for each S. In other words, we determined the visual angle that an object of the size designated by $S$ would subtend at the distance specified by $S$. To obtain these visual angle equivalents we simply inserted the metric values of S's judgments into the standard formula for computing visual angle in degrees. The mean visual angle equivalents were 0.351 , $1.24,3.94$, and $6.89 \mathrm{deg}$ for the 1-, 2-, 4, and 8-deg standards, respectively.

\section{DISCUSSION}

Earlier, five alternative outcomes of this experiment were considered. The obtained results are in greatest agreement with Outcome 5. Judged size tended to vary directly with visual angle, and judged distance tended to vary inversely with visual angle. In addition, the derived values of visual angle, computed from the judgments of size and distance, varied consistently with the visual angles actually presented. This means that there was a tendency for judged size and judged distance to be related in an invariant ratio for each of the four visual angles.

What is the implication of these findings for the question under consideration? This will depend on the construction that is placed on the distance data. Our interpretation is based on two suppositions: (1) In the present experiment, judged distance tended to vary directly with true distance. Our contention is that this correspondence is due to our procedure for varying visual angle. Our earlier experiments (Epstein \& Landauer, 1969) strongly imply that comparable distance judgments would have been obtained in response to the same variations of visual angle produced by varying the size of a target presented at a constant distance. But in this latter case, judged distance would have been unrelated to true distance. (2) The finding that the size judgments tended to be increasingly "veridical" as visual angle increased can be disregarded. There is good reason to believe (Epstein \& Landauer, 1969) that comparable size judgments would have been obtained if the distance of the disc had been constant and its size had been varied to produce visual angle variations. In this case, the size judgments would have represented decreasing veridicality with increases of visual angle.

These suppositions lead to the conclusion that the correct frame of reference for an analysis of the findings is the relationship between the judgments and visual angle. The relationship between the judgments and the objective size and distance, and between visual angle and the objective size and distance can be disregarded.

A hypothesis compatible with our data is that absolute variations of visual angle do directly lead to variations in perceived linear size, independently of the influence of perceived distance. And variations in the proportion of the visual field occluded by the target lead to variations of perceived distance; targets that occlude a large proportion of the total field loom near and vice versa. Since perceived distance and perceived size are presumed to be independent consequences of different aspects of visual angle, absolute and relative visual angle, the question of a size-distance paradox does not arise. The fact that the derived visual angles tend to vary systematically with the actual visual angle can be understood in the light of our hypothesis. Our experimental manipulations led to two concomitant stimulus variations: variations of absolute visual angle and variations of the proportion of the visual field occluded. Perceived size varied directly with the former variable, and perceived distance varied inversely with the latter variable. These two variables were inextricably tied in our experiment, with the result that judgments of size and distance were also correlated in an invariant reciprocal relationship. Consequently, visual angles derived from Ss' size and distance judgments tended to approximate the true visual angle of the target. The deviation of the derived angles from the actual visual angle reflects the fact that variations of perceived size and distance were not perfectly proportional to the variations of visual angle. 
Of the earlier studies of the perception of visual angle, only Gogel's (in press) study is directly comparable. Both Wallach and McKenna (1960) and Rock and McDermott (1964) used visual comparators to secure size judgments. Gogel (in press) had Ss make tactual size matches and verbal distance estimates of a single nonrepresentative target in the absence of distance cues. Visual angle variations were produced by presenting three different sized rectangles, individually, at the same distance. In agreement with our results, Gogel reported that size judgments varied directly with visual angle. But the distance judgments did not vary significantly. This outcome is consistent with the invariance hypothesis, and is inconsistent with our findings. In light of the many procedural differences between Gogel's experiment and the present one, it is not possible at present to reconcile the discrepant findings.

\section{REFERENCES}

EPSTEIN, W. Perceived distance in imagined space. Quarterly Journal of Experimental Psychology, 1967, 19, 341-343.
EPSTEIN, W., \& LANDAUER, A. A. Size and distance judgments under reduced conditions of viewing. Perception \& Psychophysics, 1969, 6, 273-275.

GOGEL, W. C. The effect of set on perceived egocentric distance. Acta Psychologica, 1968, 28, 283-292.

GOGEL, W. C. The sensing of retinal size. Vision Research, in press.

ROCK, I., \& MCDERMOTT, W. The perception of visual angle. Acta Psychologica, 1964, 22, 119-134.

WALLACH, H., \& McKENNA, V. V. On size-perception in the absence of cues for distance. American Journal of Psychology, 1960, 73, 458-460.

\section{NOTES}

1. This research was conducted during A.A.L.'s tenure at the University of Wisconsin, while on leave from the University of Western Australia. Appreciation is expressed to the Department of Psychology, University of Wisconsin, for providing the facilities, apparatus, and Ss for this research.

2. Address: Department of Psychology, University of Western Australia, Nedlands, Australia.

3. This research was supported by USPHS Research Grant No. 16390-01 from NIMH awarded to William Epstein. The authors are indebted to S. M. Ebenholtz and W. C. Gogel for valuable discussions of this work:

(Accepted for publication March 31, 1969.) 